\title{
A novel area efficient floating field limiting ring edge termination technique
}

\author{
M.M. De Souza ${ }^{\text {a }}$ J.V. Subhas Chandra Bose ${ }^{\text {a }, ~ E . M . ~ S a n k a r a ~ N a r a y a n a n ~}{ }^{\text {a,*, }}$, \\ T.J. Pease ${ }^{\mathrm{b}}$, G. Ensell ${ }^{\mathrm{b}}$, J. Humphreys ${ }^{\mathrm{b}}$ \\ ${ }^{a}$ Emerging Technologies Research Centre, SER Centre, Hawthorn Building, De Montfort University, Gateway, Leicester LE1 9BH, UK \\ b Southampton University Microelectronics Centre, Southampton University Highfields, Southampton, UK
}

Received 24 September 1999; received in revised form 10 April 2000

\begin{abstract}
In this paper, a floating ring edge termination structure using minimally sized lightly doped p-rings is proposed. A novel embodiment of the structure involves placement of shallow $\mathrm{p}^{+}$-regions offset from the centre of each of the $\mathrm{p}$-well rings to reduce peak electric field at the surface and to reduce sensitivity to oxide interface charges. The structures have been fabricated using an advanced, $2 \mathrm{kV}$ MOS-bipolar process technology. A close match between the simulated and experimental results validates the proposed structure. (c) 2000 Elsevier Science Ltd. All rights reserved.
\end{abstract}

\section{Introduction}

In recent years, a large effort has been devoted to the development of area efficient, reliable, planar edge termination techniques for MOS controlled power semiconductor devices. Commonly used field limiting ring (FLR) techniques involving heavily doped deep $\mathrm{p}^{+}$-rings (FLR) [1-7] can suffer from instabilities caused by high electric fields and oxide interface charges generated during processing. The role of surface passivation assumes significant importance as breakdown voltages can deteriorate by as much as $30 \%$ due to the creation of conductive surface channels between the rings by the interface charges. Therefore, heavily doped $\mathrm{p}^{+}$-FLR structures in conjunction with SIPOS/metal field plates are commonly used [8-10].

In this paper, a novel area efficient FLR structure employing lightly doped $\mathrm{p}$-well rings with shallow $\mathrm{p}^{+}$regions at the surface is presented. In our proposed approach, the depletion region extends into lightly doped $\mathrm{p}$-well regions to support high breakdown volt-

\footnotetext{
* Corresponding author. Tel.: +44-116-250-6158; fax: +44116-250-6158.

E-mail address: snem1@dmu.ac.uk (E.M. Sankara Narayanan).
}

ages with minimal cost to area. A novel embodiment of the structure involves placement of shallow $\mathrm{p}^{+}$-regions offset from the centre of each of the p-well rings to reduce the peak electric field at the surface and provide immunity to oxide interface charge. For comparison, structures employing standard heavily doped, deep $\mathrm{p}^{+}$rings are also considered. Simulation results validated by the experimental evidence support our approach.

\section{Structures}

Fig. 1 shows the simplified cross-section of the proposed $\mathrm{p}$-well $/ \mathrm{p}^{+}$-offset FLR structure with parameters as described in Table 1 . The structure is fully compatible to a planar MOS controlled power device process technology [11]. The concentration of the p-well is fully consistent with the parameters required for the clustered IGBT performance [11]. To provide consistency with the process conditions, the structures have been derived directly from T-SUPREM [12] and subsequently analysed using TMA MEDICI [12], using default parameters. Great care has been taken to ensure a close fit between the measured spreading resistance profile and that of the simulated profile. The junction depth of the rings is $11.2 \mu \mathrm{m}$ in all cases. Unless stated otherwise, an oxide interface charge of $1 \times 10^{11} \mathrm{~cm}^{-2}$ is assumed in all cases. 


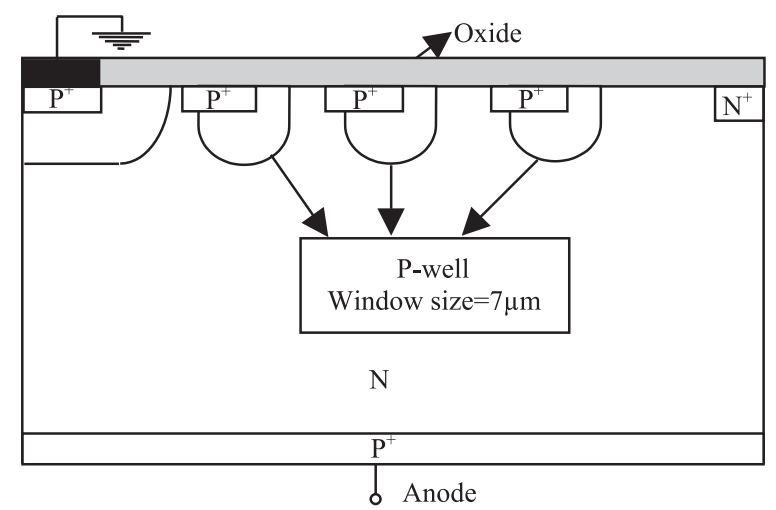

Fig. 1. A simplified cross-section of the novel FLR structure with a lightly doped $\mathrm{p}$-well and a shallow offset $\mathrm{p}^{+}$-region at the surface.

Table 1

Design parameters used for edge termination structures

\begin{tabular}{ll}
\hline Mask window size & $7 \mu \mathrm{m}$ \\
Surface concentration of p-well & $10^{16} \mathrm{~cm}^{-3}$ \\
Surface concentration of deep p $\mathrm{p}^{+}$-ring & $10^{19} \mathrm{~cm}^{-3}$ \\
and shallow $\mathrm{p}^{+}$-offset & \\
p-well and deep p $\mathrm{p}^{+}$-junction depth & $11.2 \mu \mathrm{m}$ \\
Shallow p ${ }^{+}$-offset junction depth & $1 \mu \mathrm{m}$ \\
Oxide thickness & $3 \mu \mathrm{m}$ \\
\hline
\end{tabular}

To check the validity of our approach, several highvoltage silicon planar structures have been fabricated using an advanced, $2 \mathrm{kV}$ MOS-bipolar power device technology. The $\mathrm{p}$-well region has been obtained by boron implantation of $3.5 \times 10^{13} \mathrm{~cm}^{-2}$ at $100 \mathrm{keV}$ on $250 \AA$ of oxide. The deep $\mathrm{p}^{+}$-rings have been realized by boron implantation of $1 \times 10^{16} \mathrm{~cm}^{-2}$ at $80 \mathrm{keV}$. The junction depths in both cases are identical $(11.2 \mu \mathrm{m})$. The thickness of the $\mathrm{n}$ type wafer is $400 \mu \mathrm{m}$ with a concentration of $5 \times 10^{13} \mathrm{~cm}^{-3}$. The thickness of the oxide is $3 \mu \mathrm{m}$. No passivation layers have been deposited in all cases. These FLRs are aimed for use in $2 \mathrm{kV}$ MOSbipolar devices. Therefore, to evaluate their compatibility, shallow $\mathrm{p}^{+}$-contacts have been achieved at the bottom end of the wafers. In doing so, the limitation due to the open base breakdown voltage can also be evaluated. The 'Sony Tektronix-371 A high power curve tracer' was used to check the breakdown voltage of these devices. The simulated planar breakdown voltage for the p-well structure is $2450 \mathrm{~V}$ and that of the deep $\mathrm{p}^{+}$structure is $2390 \mathrm{~V}$ and is consistent with that in the reported literature [2].

It is well known that beyond a finite number of rings, the edge termination structure does not show any increase in the breakdown voltage. Therefore, we define, for the sake of clarity, two different regions in the edge termination: (a) A linear region where the breakdown voltage can be increased by increasing the number of rings. In this case, the maximum electric field lies at the last ring.

(b) A saturation region where the addition of more rings does not change the breakdown capability. This region indicates the maximum achievable blocking voltage for the designed electric field distribution across each ring.

\section{Results and discussion}

The requirements of an edge termination structure are (a) area efficiency and (b) reliability, which depends upon the oxide interface charge. The area efficiency of a guard ring structure is strongly dependent upon the mask window size since the spacing between rings is predetermined by the desired breakdown voltage and reliability. The minimum window size is determined by the junction depth and the ratio between the breakdown voltage of a spherical or cylindrical junction to the plane parallel junction. The variation in the breakdown voltage of a lightly doped $\mathrm{p}$-well and a heavily doped $\mathrm{p}^{+}$ring, with the mask window size is illustrated in Fig. 2. In both cases, the junction depths are identical of 11.2 $\mu \mathrm{m}$ and the p-rings are connected to ground. As is evident from this figure, beyond 7 or $3 \mu \mathrm{m}$, respectively, the increase in the breakdown voltage is significantly less than the increase in the area. Therefore, a fixed mask window size of $7 \mu \mathrm{m}$ for the p-well and $3 \mu \mathrm{m}$ for the conventional $\mathrm{p}^{+}$-guard ring has been maintained in all the simulations. The mask dimension of $3 \mu \mathrm{m}$ for the deep $\mathrm{p}^{+}$-guard ring is the minimum allowable in accordance with the design rule used and is also the minimum value that has been reported in the literature so far.

The area occupied by each ring can be separated into two parts: (a) the depleted region which supports the blocking voltage and (b) the undepleted region which does not contribute to the sharing of the potential. Once

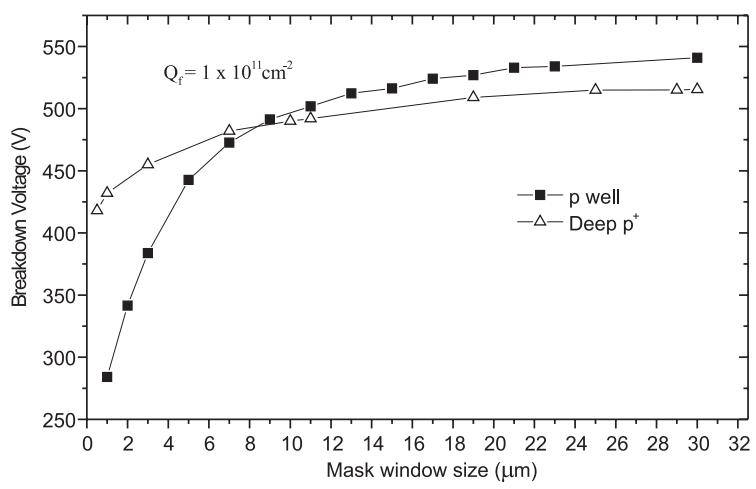

Fig. 2. The variation in the breakdown voltage with mask window size for a lightly doped p-well ring. 
the minimum dimension of the ring is established, it is possible to reduce the overall area occupied by the edge termination by reducing the concentration of the rings. In Fig. 3(a) is shown a set of results pertaining to a study of a single FLR case. As can be seen from this figure, the first ring is grounded and the next ring is floating. The objective of this study is to find the optimum distance between rings to achieve the highest blocking voltage. Three different cases have been considered: (a) $7 \mu \mathrm{m}$ lightly doped, p-well FLR, (b) a $7 \mu \mathrm{m}$, deep $\mathrm{p}^{+}$-FLR, (c) a $3 \mu \mathrm{m}$, deep $\mathrm{p}^{+}$-ring FLR and Fig. 3(b) shows the change in the breakdown voltage of the structure with the change in the distance between rings. The slope of the curve in Fig. 3(b) shows that the p-well structure is the most area efficient. Point (a) in this figure corresponds to the minimum distance possible, considering the lateral diffusions between rings. Point (b) gives the optimum distance between rings to achieve the desired, equal surface peak electric field distribution, as shown in Fig. 3(c) for all three cases, which also yields the highest breakdown voltage. The optimum distance between rings is smaller for the p-well case in comparison to that of the deep $\mathrm{p}^{+}$. In all cases, the optimum distance decreases with an increase in the positive fixed oxide charge as can be seen in Fig. 3(b). Beyond the optimum point, the electric field increases at the main junction, accompanied by a reduction in the breakdown voltage.

In an FLR involving heavily doped, deep $\mathrm{p}^{+}$-rings, the depletion region mainly extends into the lightly doped substrate. As a result, the undepleted, redundant region within each of the rings is large for a given junction depth. In the case of a lightly doped p-well (a)

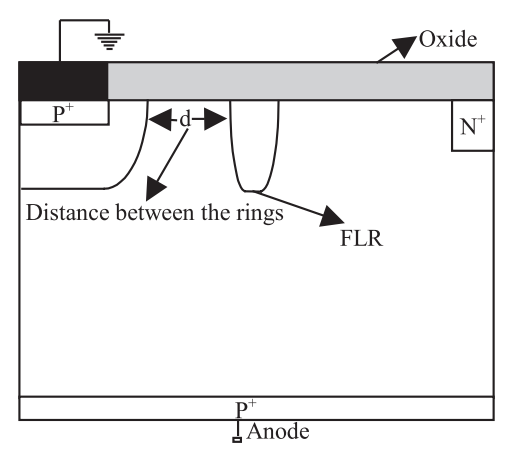

(b)

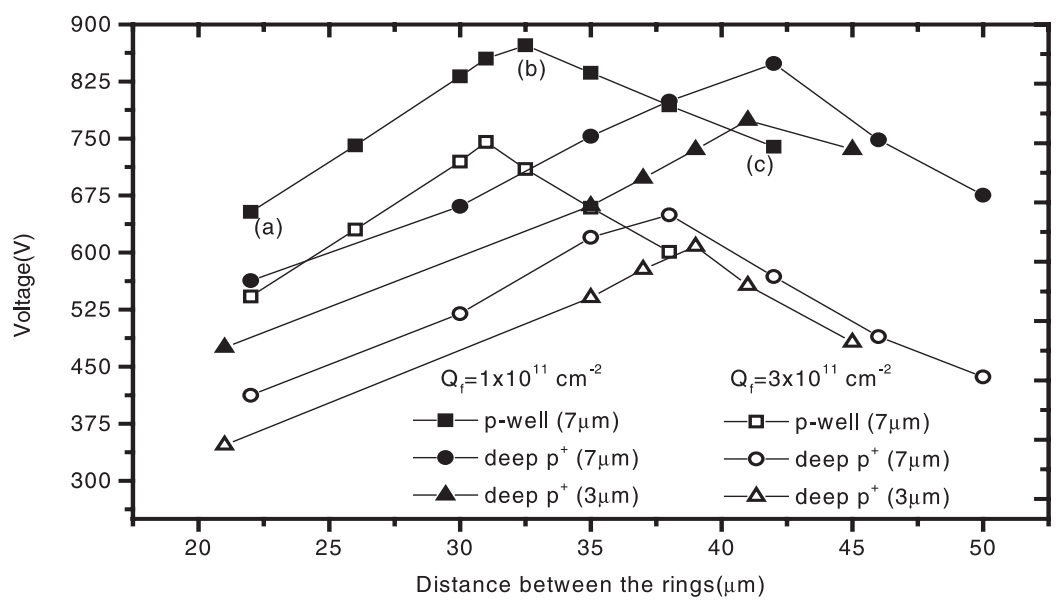

(c)

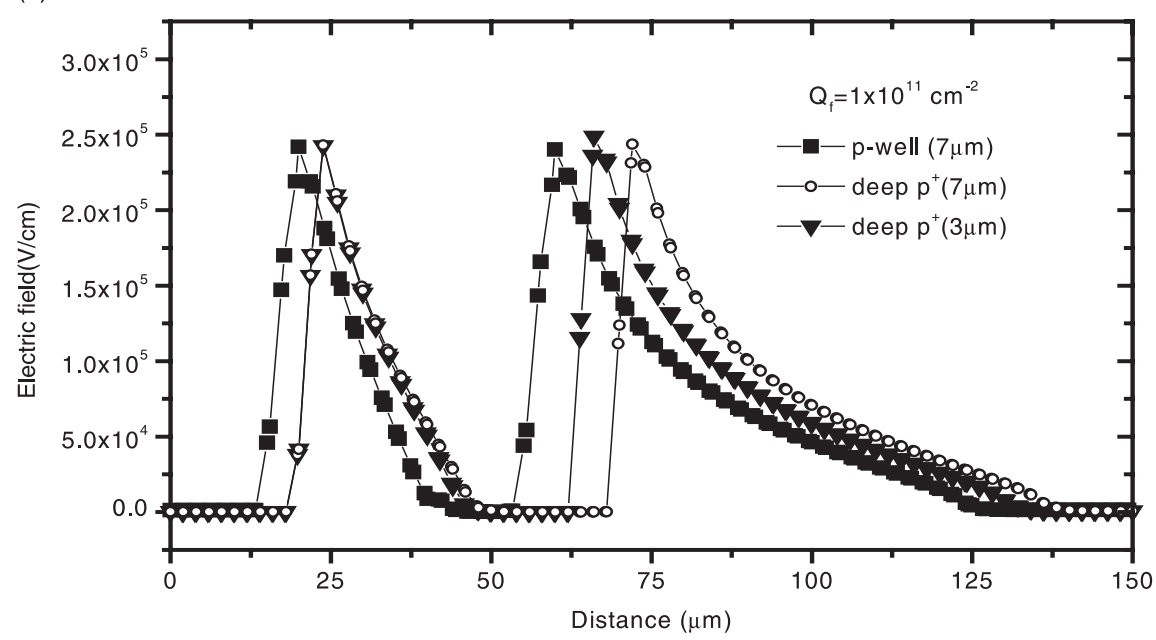

Fig. 3. (a) The simplified schematic of the single FLR study (b) the variation in the breakdown voltage with the distance between the rings and (c) electric field distribution at the optimum distance between rings. 


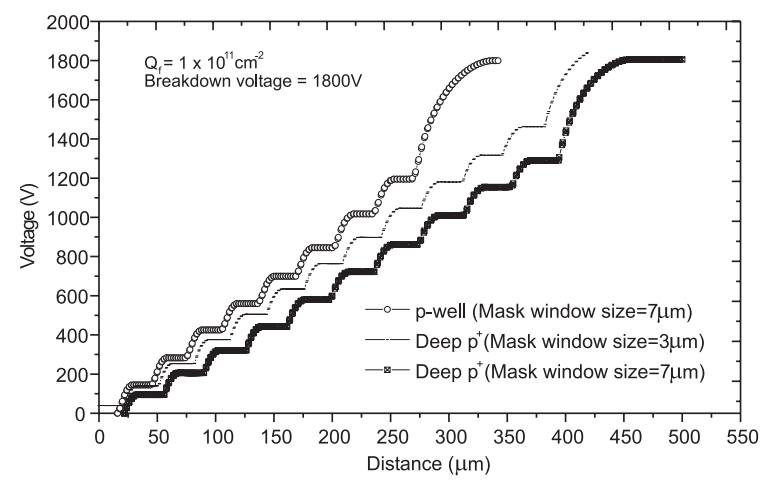

Fig. 4. The surface potential distribution of a lightly doped pwell structure and a conventional, deep $\mathrm{p}^{+}$-FLR structure at an applied voltage of $1.8 \mathrm{kV}$.

floating field ring, the depletion region extends on both sides of the junction and the potential is dropped across a larger depletion width for a given size of the ring. Therefore, the p-well FLR structure yields a smaller area in comparison to the deep $\mathrm{p}^{+}$-FLR for the same blocking voltage. This is also clearly evident from Fig. 4. This figure shows the surface potential distribution of the $\mathrm{p}$-well and the deep $\mathrm{p}^{+}$-FLR structures with spacing between rings optimized separately in each case to achieve a peak surface electric field of $1.6 \times 10^{5} \pm$ $0.1 \times 10^{5} \mathrm{~V} \mathrm{~cm}^{-1}$ at each but the last ring. It is important to note that, as a result of wider depletion region, more voltage is dropped across the lightly doped ring to achieve the same peak electric field value. As can be seen, the spreading of the depletion region into the pwell enables the area of the device to be reduced by $100 \mu \mathrm{m}$ in comparison to the deep $\mathrm{p}^{+}$-structure, even with a $3 \mu \mathrm{m}$ mask window, for an applied voltage of $1800 \mathrm{~V}$. In both cases, the structures are designed in the linear region, clearly evident from the fact that the electric field is highest at the last ring. It is possible to increase the breakdown voltage performance of the lightly doped $\mathrm{p}$-well structure even further by reducing its concentration and depth. However, the p-well forms an active device layer in a clustered insulated gate bipolar transistor and therefore, its concentration and depth are limited by the device requirements [11]. Therefore, it is not possible to reduce the concentration even further without adding another mask layer. Furthermore, the low doping of the p-well leads to the depletion at the surface, which makes the structure sensitive to fixed oxide charge.

The addition of a shallow $\mathrm{p}^{+}$-offset region to the lightly doped p-well structure results in about $250 \mathrm{~V}$ increase in the breakdown voltage as shown in Fig. 5. This is because the $\mathrm{p}^{+}$-offset region reduces the designed peak electric field at the surface. The location of the $\mathrm{p}^{+}$offset with respect to the centre of the p-well has a direct

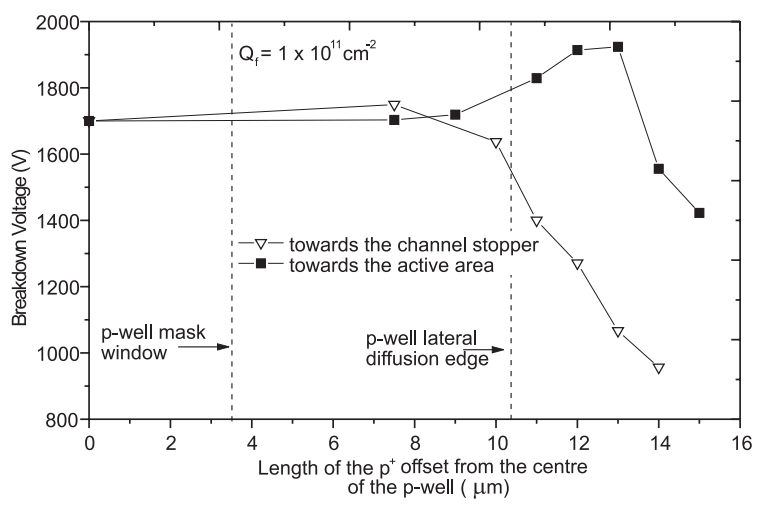

Fig. 5. The variation in the breakdown voltage with the location of the $\mathrm{p}^{+}$-offset region with respect to the centre of the $\mathrm{p}$ well ring.

influence on the breakdown voltage as clearly evident from Fig. 5. This is because once the $\mathrm{p}^{+}$-region is extended towards the main junction, the punch-through voltage is reduced. As a result, the breakdown voltage ability increases. However, increasing the length of the $\mathrm{p}^{+}$-offset beyond the optimum length such that it touches the adjacent ring results in premature electric field crowding at the last ring with an accompanying reduction in breakdown voltage. In contrast, increasing the $\mathrm{p}^{+}$-offset towards the edge of the device deteriorates the breakdown voltage significantly, as expected. If the shallow $\mathrm{p}^{+}$-region is located so as to cover the entire surface of the p-well, the peak electric field is further reduced $\left(\mathrm{p}^{+}\right.$-center in Fig. 5) resulting in area inefficiency. A comparison of the measured blocking voltage characteristics of the p-well and $\mathrm{p}$-well $/ \mathrm{p}^{+}$-offset FLRs reveal a high leakage current in the case of the p-well FLR because of the surface depletion of the lightly doped rings. However, the high leakage current does not result in a premature open-base breakdown; the structure exhibits avalanche limited breakdown characteristic. The p-well FLRs with optimally located shallow $\mathrm{p}^{+}$-offset region exhibit a significantly reduced leakage current.

In Fig. 6 is shown the variation in the breakdown voltage as a function of the number of rings in the case of deep $\mathrm{p}^{+}$-, $\mathrm{p}$-well and $\mathrm{p}$-well $/ \mathrm{p}^{+}$-offset FLR structures. The experimentally obtained results are also shown in the same figure. As defined earlier, it is apparent from the figure that the curve is characterized by both the linear and saturation regions. In the linear region, for a given applied voltage equal to or lower than the breakdown voltage, the peak electric field at each but the last ring is constant. In the saturation region, the peak electric field is uniform across all rings. Any further increase in the number of rings does not change the breakdown voltage because the depletion does not extend to those rings. Therefore, the saturation region 


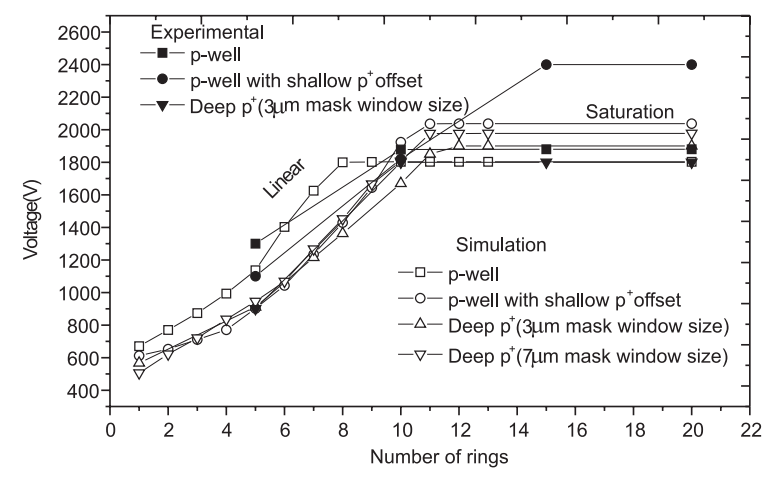

Fig. 6. The variation in the breakdown voltage with the number of rings of different FLRs.

represents the efficiency of the edge termination with respect to the maximum blocking voltage capability for the designed electric field distribution across each ring. Reducing the peak electric field at each ring and adding more rings can increase the saturation voltage achievable even further at the expense of area. This is clear from Fig. 7, which shows the distance required for each FLR to support the required blocking voltage. As can be seen from this figure, the measured results are consistent with the simulated results. Moreover, it is clear that while the p-well FLR is the most area efficient, the FLR with the $\mathrm{p}^{+}$-offset region yields the highest blocking ability in the saturation region. In the linear region, however, the p-well structure yields the highest blocking voltage. To yield a blocking voltage of $1800 \mathrm{~V}$, the distances required from the main junction edge to the edge of the last ring are 320, 380 and $420 \mu \mathrm{m}$ for the p-well, $\mathrm{p}^{+}$-offset and the deep $\mathrm{p}^{+}$-ring FLRs, respectively.

The influence of the positive fixed oxide interface charge on the breakdown voltage is strongly influenced upon whether the structure is designed in the linear or saturation region. In the linear region, the electric field across each ring is significantly less than the critical

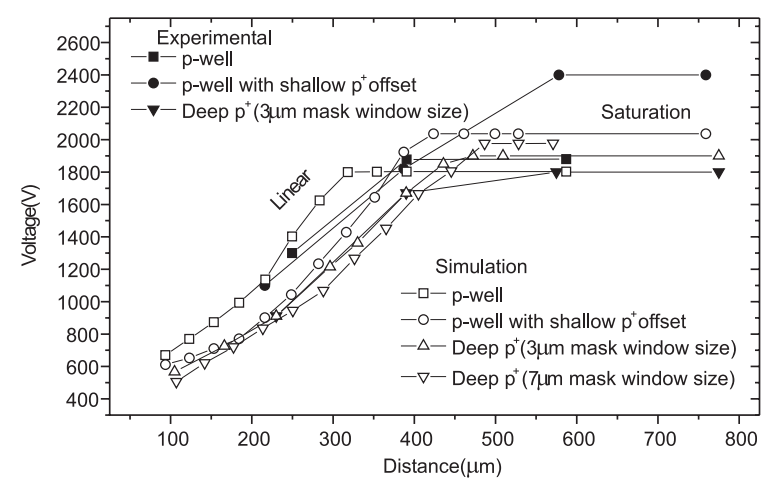

Fig. 7. The variation in the breakdown voltage with distance. The distance increases as the number of rings is increased. electric field with the maximum value at the last ring. The positive fixed charge underpins the depletion region and results in an increase in the electric field. This results in an increase in the breakdown voltage. In the linear region, the shallow $\mathrm{p}^{+}$-structure shows a $10 \%$ increase in the breakdown voltage and a $7 \mu \mathrm{m}$ deep $\mathrm{p}^{+}$shows a $4.5 \%$ increase in the breakdown voltage with changes in the fixed oxide charge from $5 \times 10^{10}$ to $4.5 \times 10^{11} \mathrm{~cm}^{-2}$. However, in the saturation region, the influence of the fixed oxide charge is such that it causes an imbalance in the electric field distribution. Therefore, in all cases, the breakdown voltage is deteriorated in the saturation region. The shallow $\mathrm{p}^{+}$-offset and $7 \mu \mathrm{m}$ deep $\mathrm{p}^{+}$-structure shows about $10 \%$ reduction in the breakdown voltage with changes in the fixed oxide charge from $5 \times 10^{10}$ to $4.5 \times 10^{11} \mathrm{~cm}^{-2}$. The lightly doped p-well FLR is most sensitive to oxide interface charge, whether designed in the linear region or saturation regions. This structure shows about $23 \%$ reduction in the breakdown voltage in the saturation region and this value reduces to $12 \%$ in the linear region.

The benefits of the presence of positive charges at the interface to the breakdown voltage capability of a single, deep $\mathrm{p}^{+}$-ring are well established [13]. Our results show, for the first time, that the fixed oxide charge sensitivity of a deep $\mathrm{p}^{+}$-structure is also dependent upon the mask window size. For a $3 \mu \mathrm{m}$ based deep $\mathrm{p}^{+}$-structure, the interface charge from $5 \times 10^{10}$ to $4.5 \times 10^{11} \mathrm{~cm}^{-2}$ reduces the breakdown voltage by $13 \%$ irrespective of whether the structure is in the linear or in the saturation region. This is because the influence of the cylindrical curvature effect dominates the breakdown characteristics in a manner similar to a p-well FLR. When the mask window size is increased to $7 \mu \mathrm{m}$ the influence of the positive fixed oxide charge on the breakdown voltage decreases in the saturation region.

While the conventional FLR structure has been considered prone to oxide interface charge, this has been traditionally overcome with the use of metal field plates. The role of the metal field plate in the edge termination structure is to reduce the peak electric field at each ring and to push the peak electric field towards the last ring [7]. The $\mathrm{p}^{+}$-offset structure designed in the linear region uses a similar principle, where the $\mathrm{p}^{+}$-regions at the surface reduce the peak electric field at each ring and ensures that the peak electric field remains at the last ring in the presence of oxide interface charge. The sensitivity of the metal field plate structure on the intervening oxide layer is effectively overcome by the $\mathrm{p}^{+}$-offset structure.

In any edge termination, the variation in the resistivity of the substrate can influence the breakdown voltage capability of the device. A study of the breakdown voltage with a $\pm 20 \%$ variation in the substrate concentration of the structures in the linear region reveals a $4.5 \%$ increase in the breakdown voltage of the 
$\mathrm{p}^{+}$-offset and deep $\mathrm{p}^{+}$-structures. In comparison, the lightly doped p-well structure shows $6.4 \%$ increase in the breakdown voltage. The breakdown voltage increases with an increase in the n-type concentration as the enhanced surface doping serves to increase and control the punch-through voltage between the rings as described in Ref. [14].

The technique proposed herein is applicable to higher blocking voltages as well. With the increase in the voltage rating, the variation in the substrate resistivity is high. Therefore, to accommodate the variations, the mask window size increases very slightly. For example, for a $3.8 \mathrm{kV}$ non-punch-through technology, the mask window size required is $10 \mu \mathrm{m}$ and for $6.5 \mathrm{kV}$, it is 11 $\mu \mathrm{m}$. The total distance required to support $3.8 \mathrm{kV}$ is between 800 and $1000 \mu \mathrm{m}$, and for $6.5 \mathrm{kV}$, it is about 2$2.5 \mathrm{~mm}$. At these high voltage ranges, the crystalline defect densities and the surface charge density values can no longer be ignored against low background doping concentration. Therefore, great care has to be taken to achieve stability in any edge termination structure. To achieve the stability, the electric field across the rings has to be reduced significantly at the expense of the overall area of edge termination.

\section{Conclusions}

In this paper, a novel edge termination structure comprising of lightly doped $\mathrm{p}$-well rings with enhanced $\mathrm{p}^{+}$-doping at the surface has been analyzed in detail. The $\mathrm{p}^{+}$-regions are placed at an offset with respect to the centre of the ring. The FLR is found to yield the maximum breakdown voltage of $2.4 \mathrm{kV}$ with an area smaller than that of the conventional $\mathrm{p}^{+}$-FLR. This is because the proposed structure has inherent benefits of area savings realized by the use of lightly doped p-well rings and charge insensitivity due to its highly doped, $\mathrm{p}^{+}$offset surface region.

\section{Acknowledgements}

This work is supported by EPSRC grant GR/L27248. The authors would like to thank Westcode Semiconductors Ltd., for their constant support in the project and for providing the wafers and access to their facilities to carry out this work.

\section{References}

[1] Adler MS, Temple VAK, Ferro AP, Rustay RC. IEEE Trans Electron Dev 1977;ED-24:107.

[2] Baliga BJ. IEE Proc 1982;129:173.

[3] Byrne DJ, Towers MS, Board K. IEE Proc 1991;138:109.

[4] Ma TS, Grabowski WB. Solid State Electron 1992;35:201.

[5] Tang BQ, Gao YM, Luo JS. Solid State Electron 1997;41:1821.

[6] Macary V, Charitat G, Bafleur M, Buxo J, Rossel P. Proc Int Symp Power Semicond Dev \& ICs. 1992. p. 230-3.

[7] Yilmaz H. IEEE Trans Electron Dev 1997;38:1666.

[8] Saitoh R, Nishiura A, Sakurai K. Proc 1992 ISPSD Tokyo. p. 206.

[9] Basavana Goud C, Bhat KN. IEEE Trans Electron Dev 1994;41:1856.

[10] Jaume D, Charitat G, Reynes JM, Rossel P. IEEE Trans Electron Dev 1991;38:1681.

[11] Narayanan EMS, Sweet M, Spulber O, De Souza MM. Phys Semicond Dev, vol. II. Allied Publishers, 1999. ISBN 81-7023-998-2, p. 1307-12.

[12] TSUPREM IV and MEDICI manuals, Avanti Associates Inc.

[13] Baliga BJ. Modern Power Device New York: WileyInterscience, 1987. p. 95.

[14] Clark LE, Davies RB, Groenig PJ. Proc ISPSD'92, Tokyo. p. 86. 